\title{
Fatigue crack initiation and damage mechanisms during ultrasonic fatigue testing of cast aluminium alloy AISi7Mg
}

\author{
Dominik Krewerth ${ }^{\mathrm{a}}$, Anja Weidner and Horst Biermann \\ TU Bergakademie Freiberg, Institute of Materials Engineering, Gustav-Zeuner-Straße 5, \\ 09599 Freiberg, Germany
}

During the last years, an increased interest in in situ-damage characterization during ultrasonic fatigue testing evolved. Different non-contact in situ-surveillance systems have been developed or applied to the present ultrasonic fatigue testing systems, e.g. analysis of the nonlinearity parameter and the resonant frequency [1, 2] as well as thermal measurements [3-5]. In addition, Heinz et al. [6] performed noncontact strain measurements during ultrasonic fatigue testing.

The aim of the present investigations is to evaluate the fatigue crack initiation in the cast aluminium alloy AlSi7Mg (A356) in sand and die cast condition under high cycle and very high cycle fatigue loading conditions. Therefore, a combined analysis of the development of the specimen temperature, the resonant frequency $\mathrm{f}_{\text {res }}$ and the nonlinearity parameter $\beta_{\text {rel }}$ was performed. For such investigations it is essential to keep the ambient temperature strictly constant and the increase of temperature due to the resonant conditions as low as possible. Therefore, a spot cooler using a vortex tube was applied to the ultrasonic fatigue testing system. With this special cooling device it was possible to keep the ambient temperature constant. An infrared thermo-camera system was used for the in situ-examination of the specimen temperature and the surveillance of the ambient temperature during ultrasonic fatigue testing. The determination of the resonant frequency and the nonlinearity parameter was realized online during fatigue tests according to Mayer et al. [2]. After failure, the fracture surfaces of the specimens were investigated intensively by scanning electron microscopy (SEM). According to Krewerth et al. [5], the correlation of the results from thermographic and fractographic investigations can shed some light on the crack initiation point.

Figure 1a shows the $\mathrm{S}-\mathrm{N}$ curves of the fatigue tests on the aluminum specimens produced by sand casting (batch 1) and die-casting (batch 2) in hot isostatically pressed condition, respectively. Specimens of both batches failed due to casting porosity. However, the casting porosity in the sand cast material was significantly higher leading to a lower fatigue strength. Several specimens were investigated by thermographic measurements accompanied by simultaneous analysis of the resonant frequency as well as the nonlinearity parameter. Exemplarily, specimen 1 (sand casting, see Fig. 1a) is explained here in more detail. The development of the resonant frequency $\left(\mathrm{f}_{\text {res }}\right)$, the nonlinearity parameter $\left(\beta_{\text {rel }}\right)$, the ambient temperature $\mathrm{T}_{\mathrm{amb}}$, the maximum temperature $\mathrm{T}_{\max }$ and the temperature variation $\Delta \mathrm{T}$ is shown in fig. 1b. This specimen failed due to an internal defect at $\mathrm{N}_{\mathrm{f}}=2.16 \times 10^{6}$ cycles. The dashed black

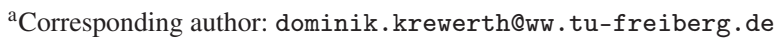

This is an Open Access article distributed under the terms of the Creative Commons Attribution License 4.0, which permits unrestricted use, distribution, and reproduction in any medium, provided the original work is properly cited. 

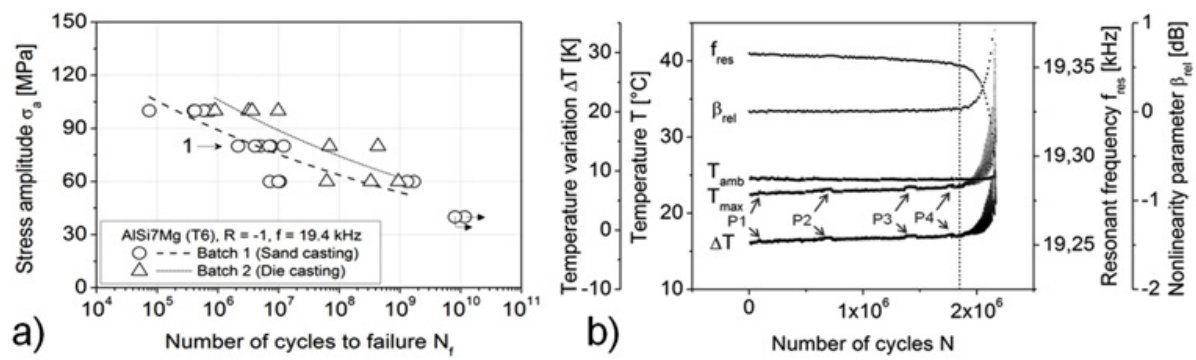

Figure 1. a) Stress vs. number of cycles to failure ( $\mathrm{SN})$ - plot of the investigated $\mathrm{Al}$ alloy and b) development of temperature $\left(\mathrm{T}_{\max }, \mathrm{T}_{\mathrm{amb}}, \Delta \mathrm{T}\right)$, resonant frequency $\left(\mathrm{f}_{\mathrm{res}}\right)$ and nonlinearity parameter $\left(\beta_{\text {rel }}\right)$ of specimen 1.
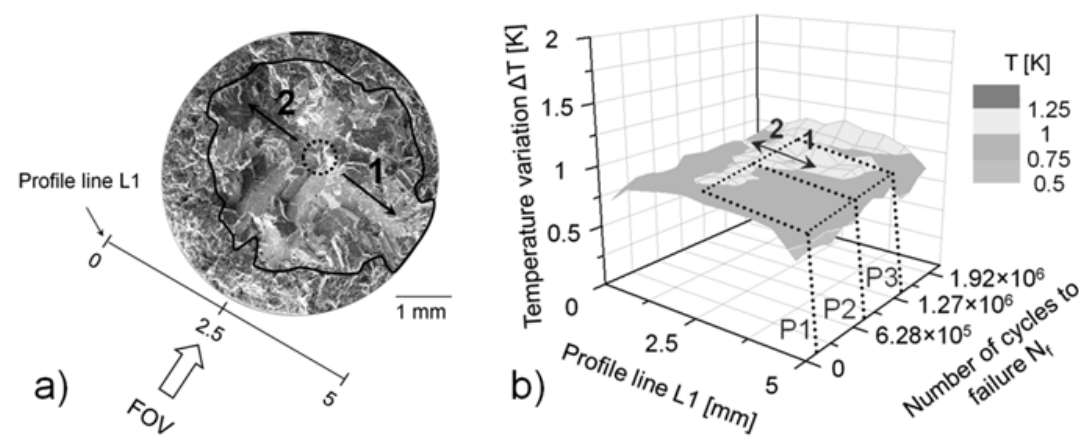

Figure 2. a) Correlated thermography and fractography. a) Fracture surface of specimen 1. b) Temperature distribution during fatigue testing of specimen 1 along a profile line marked in a).

line in fig. $1 \mathrm{~b}$ illustrates the point of final crack growth simultaneously observed by the four parameters $\Delta \mathrm{T}, \mathrm{T}_{\max }, \mathrm{f}_{\text {res }}$ and $\beta_{\text {rel }}$, respectively. Obviously, the development of all parameters is independent from the ambient temperature $T_{a m b}$ measured in the vicinity of the gauge length of the specimen (see $T_{a m b}$ in fig. 1b). However, four discontinuities were observed in the development of $\mathrm{T}_{\max }$ as well as of $\Delta \mathrm{T}$ at $1.2 \times 10^{5}$ cycles $(\mathrm{P} 1), 7 \times 10^{5}$ cycles $(\mathrm{P} 2), 1.35 \times 10^{6}$ cycles $(\mathrm{P} 3)$ and $1.75 \times 10^{6}$ cycles $(\mathrm{P} 4)(\mathrm{see}$ Fig. 1b). In addition, a detailed analysis of the fracture surface in correlation with the temperature development at the crack initiation point for this specimen was performed.

Figure 2a shows the fracture surface showing a casting pore as the crack initiation point marked by a dashed black circle. The black arrows $(1,2)$ indicate two directions of crack growth. In addition, the field of view (FOV) of the thermo-camera is indicated. A horizontal temperature line along the whole cross section of the specimen (profile line L1) was evaluated in order to obtain more detailed two-dimensional information on the temperature development at the crack initiation point and about the crack propagation. The results are shown Fig. 2b. The three discontinuities P1, P2, P3 detected in Fig. 1b are marked by dashed black lines.

The evolution of the horizontal temperature profile shown in Fig. $2 b$ and the points of discontinuity denoted in Fig. 1b by black arrows are well correlated to the fracture surface shown in Fig. 2a. The first local temperature spot is observed during the first stage of ultrasonic fatigue testing at $1.2 \times 10^{5}$ cycles (cf. Figs. $1 \mathrm{~b}$ and $2 \mathrm{~b}$ ), thus indicating probably the point of crack initiation. An extension of the temperature peak towards the right side of the specimen is observed at $7 \times 10^{5}$ cycles in Fig. $2 \mathrm{~b}$. This extended temperature field is well correlated to the crack path denoted by arrow number 1 in Fig. $2 \mathrm{a}$ and explains the second point of discontinuity illustrated in Fig. 1b. A more pronounced increase in temperature is observed at $1.35 \times 10^{6}$ cycles on both crack directions (see arrows 1 and 2 in Fig. 2). 


\section{References}

[1] A. Kumar, R. Adharapurapu, W. Jones, T. Pollock, Scripta Mater. 64 (2011) 65-68

[2] H. Mayer, M. Fitzka, R. Schuller, Ultrasonics 53 (2013) 1425-1432

[3] D. Wagner, N. Ranc, C. Bathias, P. C. Paris, Fatigue Fract. Eng. M. 33 (2009) 12-21

[4] H. Xue, D. Wagner, N. Ranc, E. Bayraktar, Fatigue Fract. Eng. M. 29 (2006) 573-580

[5] D. Krewerth, A. Weidner, H. Biermann, Ultrasonics 53 (2013) 1441-1449

[6] S. Heinz, F. Balle, G. Wagner, D. Eifler, Ultrasonics 53 (2013) 1433-1440 